\title{
CUANDO LA VIOLENCIA SOBRA Y EL TRABAJO FALTA. ESCENARIOS PARA JÓVENES DE SECTORES POPULARES URBANOS EN AMÉRICA LATINA
}

\author{
JUAN PABLO PÉREZ SÁINZ ${ }^{1}$
}

\begin{abstract}
RESUMEN
El International Development Research Center/Centre de Recherches pour le Développement International (IDRC/CRDI) de Canadá ha auspiciado $y$ financiado un conjunto de investigaciones, coordinadas por FLACSO Costa Rica, que han intentado articular tres problemáticas: jóvenes de sectores populares urbanos, violencias y oportunidades laborales. El presente texto sistematiza este conjunto de estudios, proponiendo un marco analítico para abordar la interacción entre violencia y trabajo y el accionar de las/los jóvenes. A partir del mismo se identifican cuatro escenarios de agencia juvenil en este tipo de contextos donde la violencia sobra y el trabajo falta. El texto concluye con una serie de interrogantes que la actual pandemia plantea a estos escenarios y que perfilan problemáticas de investigación de cara al futuro inmediato.
\end{abstract}

PALABRAS CLAVES: JÓVENES DE SECTORES POPULARES, VIOLENCIAS, TRABAJO

\footnotetext{
${ }^{1}$ Investigador de FLACSO Costa Rica. Correo electrónico: jpps@flacso.or.cr
} 


\title{
QUANDO A VIOLÊNCIA É DEMASIADA E O TRABALHO É MUITO POUCO. CENÁRIOS PARA JOVENS DE SETORES POPULARES URBANOS NA AMÉRICA LATINA
}

\begin{abstract}
RESUMO
O International Development Research Center/Centre de Recherches pour le Développement International (IDRC/CRDI) do Canadá patrocinou e financiou uma série de pesquisas, coordenadas pela FLACSO Costa Rica, que tentaram articular três temas: jovens de setores populares urbanos, violências e oportunidades de trabalho. Este texto sistematiza este conjunto de estudos ao propor um referencial analítico para abordar a interação entre a violência, o trabalho e as ações das e dos jovens. Com base nisso, quatro cenários de agência juvenil são identificados neste tipo de contextos em que a violência é demasiada e o trabalho é muito pouco. O texto conclui com uma série de perguntas que a atual pandemia apresenta a estes cenários e que esboçam temas de pesquisa para o futuro imediato.
\end{abstract}

PALAVRAS-CHAVE: JOVENS DE SETORES POPULARES, VIOLÊNCIAS, TRABALHO

\section{WHEN VIOLENCE IS TOO MUCH AND WORK LACKS. SCENARIOS FOR YOUNG PEOPLE FROM WORKING-CLASS URBAN COMMUNITIES IN LATIN AMERICA}

\begin{abstract}
The International Development Research Center/Centre de Recherches pour le Développement International (IDRC/CRDI) of Canada has sponsored and financed a series of research studies, coordinated by FLACSO Costa Rica, which have attempted to assemble three issues: young people from working-class urban communities, violence, and job opportunities. This text systematizes this set of studies by proposing an analytical framework to address the interaction between violence and work, and the actions of male and female young people. Based on it, four scenarios of youth agency are identified in this type of contexts where violence is too much, and work lacks. The text concludes with a series of questions that the current pandemic poses to these scenarios and that outline research issues for the immediate future.
\end{abstract}

KEYWORDS: YOUNG PEOPLE FROM WORKING-CLASS COMMUNITIES, VIOLENCE, WORK 


\section{INTRODUCCIÓN}

En la región existe una copiosa bibliografía sobre jóvenes y violencias y sobre jóvenes y trabajo, pero son pocos los intentos de articular estas tres problemáticas $^{2}$. El presente texto tiene como objetivo explorar los nexos entre estas tres cuestiones, a partir de la identificación de escenarios en los que actúan jóvenes provenientes del mundo popular urbano. Esta reflexión es la culminación de un conjunto de investigaciones en la región en las que FLACSO Costa Rica, y en concreto el autor de este texto, ha estado involucrado gracias al auspicio y financiamiento del International Development Research Center/Centre de Recherches pour le Développement International (IDRC/CRDI) de Canadá.

El presente texto inicia con una nota metodológica donde se identifican las distintas investigaciones que han provisto la evidencia empírica en la cual se sustenta la propuesta analítica de los escenarios. En el segundo apartado, el central, se argumenta la construcción de estos, a partir de la interacción entre violencia y trabajo y el accionar de las/los jóvenes en ellos. Se concluye con una serie de interrogantes que la actual pandemia plantea a estos escenarios y que perfilan problemáticas de investigación de cara al futuro inmediato.

\section{LAS INVESTIGACIONES DE BASE: NOTA METODOLÓGICA}

El primer antecedente que reseñar es la investigación llevada a cabo en asentamientos populares urbanos en las áreas metropolitanas de San José (Costa Rica) y San Salvador (El Salvador). El objetivo de este estudio era identificar trayectorias de jóvenes a partir de las mediaciones (familia, escuela, trabajo, instituciones, territorio y pares) que moldean su acción (Pérez Sáinz, 2018). Con

\footnotetext{
${ }^{2}$ Agradecemos los comentarios, a un borrador de este texto, de Milena Arancibia, María del Pilar Castillo Valencia y José Antonio Espinal Betanzo.
} 
base en estos resultados se concibió la iniciativa regional «Vidas Sitiadas I», dentro de la cual se hizo un primer intento por identificar campos de articulación entre violencia y trabajo ${ }^{3}$. Este intento tuvo dos componentes. El primero consistió en el aporte de una serie de estudios sobre temas específicos considerados claves: construcción de masculinidades de jóvenes en contextos de marginación social (Cruz Sierra, 2019); involucramiento de jóvenes en pandillas y crimen organizado (Castillo Valencia, Salazar Trujillo y Caicedo Hurtado, 2019; López Ramírez, 2019); participación de jóvenes en iniciativas de economía social y popular (Miranda Rodríguez, 2019); y jóvenes en empleos globalizados (el caso de los call centers) (Hualde Alfaro, 2019). El segundo componente radicó en una revisión de las bibliografías recientes en América Latina sobre juventud y violencias, por un lado, y sobre juventud y trabajo, por otro lado (Pérez Sáinz, 2019). En este último trabajo se ha buscado la identificación de campos de articulación entre violencia y trabajo y dentro de ellos los factores críticos que podían afectar las acciones de jóvenes de sectores populares.

Un primer campo es el doméstico, donde hay que destacar, por un lado, la existencia de mujeres jóvenes recluidas en este espacio que estarían disponibles para incorporarse al mercado laboral, pero que están condenadas a la realización de trabajo doméstico; esta condición se viabiliza por su invisibilización. Por otro lado, se debe mencionar la construcción de masculinidades ante un nuevo mundo laboral, donde el papel tradicional del hombre como proveedor del hogar se dificulta. Referentes de violencia emergen como sustitutos en esos procesos de configuración identitaria. El segundo campo es el territorial, que conlleva cuatro factores críticos articuladores: las trabas a la movilidad laboral existente en territorios controlados por actores violentos; la estigmatización que padecen los pobladores de estos territorios por la metonimia a la que se les somete y que les atribuye, de manera esencialista, la naturaleza

\footnotetext{
${ }^{3}$ Hay una página web de esta iniciativa regional: https://www.vidasitiadas.com/
} 
delictiva de los actores violentos, afectando de manera especial a jóvenes en la búsqueda de trabajo; la oferta laboral desde las organizaciones delictivas a jóvenes de sectores populares; y la existencia de extorsiones por actores violentos que controlan esos territorios hacia actividades autogeneradas que las hacen inviables. El tercer campo es el del consumismo, donde la insuficiencia de ingresos laborales combinada con presiones consumistas puede llevar a que haya acciones ocasionales de transgresión que pueden degenerar en actos delictivos (Pérez Sáinz, 2019).

Además, Vidas Sitiadas ha agrupado a un conjunto de investigaciones que han abordado cuestiones variadas en países diferentes. El cuadro 1 muestra las instituciones ejecutoras de estos estudios y las problemáticas de investigación que se han planteado. 


\section{CuAdro 1. ProblemáticAs de INVESTIGACIÓN DE PROYECTOS DE VidAS} SITIADAS I

\begin{tabular}{|c|c|c|}
\hline Institución & Proyecto & $\begin{array}{l}\text { Problemática de } \\
\text { investigación }\end{array}$ \\
\hline $\begin{array}{l}\text { Universidad del } \\
\text { Valle, Cali } \\
\text { (Colombia) }\end{array}$ & $\begin{array}{l}\text { Estrategias de inclusión } \\
\text { laboral y social de jóvenes } \\
\text { en zonas marginadas de } \\
\text { Cali, Colombia }\end{array}$ & $\begin{array}{l}\text { Caracterización de los } \\
\text { jóvenes del Programa } \\
\text { Tratamiento Integral de } \\
\text { Pandillas-Jóvenes Sin } \\
\text { Fronteras (TIP-JSF) y } \\
\text { análisis de las estrategias de } \\
\text { estos jóvenes por insertarse } \\
\text { en el mercado laboral de } \\
\text { Cali. }\end{array}$ \\
\hline $\begin{array}{l}\text { Fundación Salvador } \\
\text { del Mundo } \\
\text { (FUSALMO) (El } \\
\text { Salvador) }\end{array}$ & $\begin{array}{l}\text { Los empresarios frente al } \\
\text { empleo de jóvenes } \\
\text { residentes en contextos } \\
\text { violentos en El Salvador: un } \\
\text { análisis de trayectorias } \\
\text { sociolaborales y cultura } \\
\text { empresarial }\end{array}$ & $\begin{array}{l}\text { Reconstrucción de } \\
\text { trayectorias sociolaborales } \\
\text { de jóvenes beneficiarios del } \\
\text { Programa de Gestión } \\
\text { Sociolaboral 2013-2017 } \\
\text { (proyecto «Jóvenes creando } \\
\text { futuro»). }\end{array}$ \\
\hline $\begin{array}{l}\text { Confederación } \\
\text { Patronal de la } \\
\text { República Mexicana- } \\
\text { León y la Secretaría } \\
\text { de Educación Pública } \\
\text { (COPARMEX/SEP) }\end{array}$ & $\begin{array}{l}\text { La educación dual en } \\
\text { México: generación de } \\
\text { oportunidades laborales para } \\
\text { jóvenes de extracción } \\
\text { popular en el Estado de } \\
\text { Guanajuato }\end{array}$ & $\begin{array}{l}\text { Analizar la aplicación del } \\
\text { Modelo Mexicano de } \\
\text { Formación Dual (MMFD), } \\
\text { tanto en las empresas como } \\
\text { en los centros educativos, e } \\
\text { identificar sus efectos en las } \\
\text { trayectorias laborales y de } \\
\text { vida de los denominados } \\
\text { aprendices duales. }\end{array}$ \\
\hline $\begin{array}{l}\text { Fundación } \\
\text { Salvadoreña para el } \\
\text { Desarrollo } \\
\text { Económico y Social } \\
\text { (FUSADES) (El } \\
\text { Salvador) } \\
\end{array}$ & $\begin{array}{l}\text { Fortaleciendo medios de } \\
\text { vida y reduciendo la } \\
\text { violencia urbana a través de } \\
\text { la inclusión de jóvenes y la } \\
\text { innovación social (Proyecto } \\
\text { Núcleo de Glasswing) }\end{array}$ & $\begin{array}{l}\text { Identificar los estigmas que } \\
\text { afectan a los jóvenes } \\
\text { participantes del proyecto } \\
\text { Núcleo, para registrar sus } \\
\text { causas y así poderlas superar. }\end{array}$ \\
\hline FLACSO Argentina & $\begin{array}{l}\text { COLECTIVA JOVEN } \\
\text { (Jóvenes hacen colectivo): } \\
\text { proyecto de investigación y } \\
\text { acción dirigido a apoyar } \\
\text { grupos de jóvenes orientados } \\
\text { a la producción en barrios } \\
\text { marginalizados de la } \\
\text { periferia de Buenos Aires }\end{array}$ & $\begin{array}{l}\text { Indagar las dinámicas de } \\
\text { inclusión social de las/os } \\
\text { jóvenes de COLECTIVA } \\
\text { JOVEN. }\end{array}$ \\
\hline
\end{tabular}


Como se puede apreciar se trata de cinco proyectos que cubren cuatro países, lo que permite, hasta cierto punto, realizar una reflexión de carácter regional. Por otro lado, las problemáticas se ubican entre las dos grandes cuestiones que nos competen. Así, los estudios de FUSADES y, sobre todo, los de la Universidad del Valle y FLACSO Argentina, tienen una fuerte implantación en el territorio, por lo que la problemática de las violencias se evidencia de manera más explícita. En cuanto a los otros dos hacen el recorrido desde los territorios hacia el mundo del trabajo, enfatizando la intermediación laboral sustentada en actividades de formación.

El cuadro 2 da una idea del alcance de estas investigaciones en términos de sus opciones metodológicas.

CuAdro 2. METOdOlOGías DE PROYECTOS DE VidAs Sitiadas I

\begin{tabular}{|c|c|c|}
\hline Institución & Universo de estudio & Técnicas de investigación \\
\hline Universidad del Valle & $\begin{array}{l}\text { Informe } 1: 2.414 \text { jóvenes } \\
\text { miembros de } 86 \text { pandillas } \\
\text { participantes en el programa } \\
\text { TIP-SJF } \\
\text { Informe 2: } 110 \text { jóvenes del } \\
\text { programa TIP-SJF para el } \\
\text { análisis de redes }\end{array}$ & $\begin{array}{l}\text { Encuesta de caracterización del } \\
\text { Programa TIP-JSF y grupos } \\
\text { focales } \\
\text { Utilización de fuentes } \\
\text { secundarias: encuesta del } \\
\text { SISBEN (con corte a 2018), } \\
\text { Encuesta de Empleo y Calidad } \\
\text { de Vida realizada en Cali (2012 } \\
\text { y 2013), base de datos de } \\
\text { homicidios del Observatorio } \\
\text { Social de Cali (1993-2018) y } \\
\text { cartografía } \\
\text { Encuesta de caracterización del } \\
\text { Programa TIP-JSF } \\
\text { Cuestionario para análisis de } \\
\text { redes } \\
\text { Cinco grupos focales }\end{array}$ \\
\hline FUSALMO & $\begin{array}{l}308 \text { jóvenes del proyecto } \\
\text { «Jóvenes creando futuro» entre } \\
2013 \text { y } 2017 \text { que lograron } \\
\text { obtener un trabajo } \\
44 \text { empresarios contactados para } \\
\text { encuesta en línea }\end{array}$ & $\begin{array}{l}22 \text { entrevistas en profundidad } \\
\text { Cinco respuestas }\end{array}$ \\
\hline
\end{tabular}




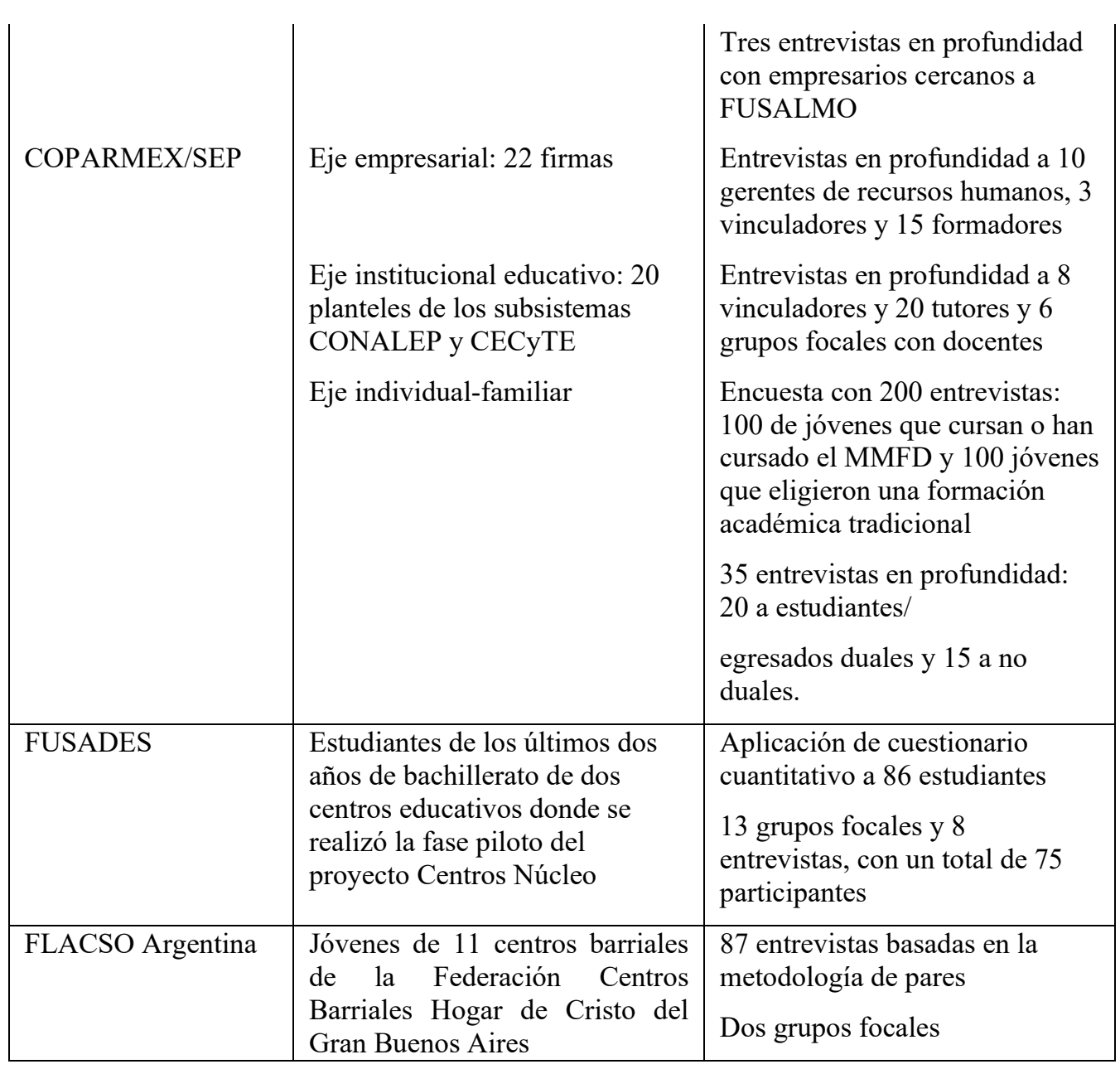

Como se puede apreciar, todas las investigaciones han tenido como universos de estudio a jóvenes de sectores populares urbanos. En algunos casos, los estudios de FUSALMO y — en especial — el de Guanajuato han ampliado el universo a empresas y a establecimientos educativos. Las técnicas utilizadas han sido variadas, pero se debe destacar la metodología utilizada en el proyecto de FLACSO Argentina por su opción por la metodología de pares.

Es importante señalar que todos estos proyectos se vieron afectados por la pandemia de la Covid-19. Esto supuso que en algunos casos no se lograran de manera plena - todos los objetivos planteados inicialmente. Al respecto destacan dos ejemplos. En el estudio de FUSALMO no se logró entrevistar en línea a suficientes empresarios para indagar sobre la problemática de la cultura 
empresarial, especialmente en relación con su visión estigmatizadora de fuerza laboral joven proveniente de territorios con presencia de pandillas violentas. También la investigación en Guanajuato no pudo llevar a cabo alguno de sus objetivos del tercer eje, referido a las trayectorias de jóvenes.

Ha sido a partir de los resultados arrojados por este conjunto de investigaciones, además de la primera llevada a cabo en Centroamérica y de la propuesta inicial de campos y factores críticos de articulación, que se ha intentado elaborar una propuesta analítica sobre escenarios para la agencia de jóvenes de sectores populares urbanos definidos por la violencia y las oportunidades laborales. Este es el contenido del siguiente apartado ${ }^{4}$.

\section{JÓVENES, VIOLENCIA Y TRABAJO: UNA PROPUESTA ANALÍTICA}

Todas las investigaciones muestran universos signados, en distinto grado, por alta violencia y pocas oportunidades de trabajo ${ }^{5}$. Estos referentes situacionales no deben olvidarse cuando se interpretan los escenarios. Es decir, se está ante realidades donde sobra violencia y falta trabajo.

Si bien en este tipo de contexto se reducen las opciones de las/los jóvenes, en la identificación de escenarios se ha querido priorizar su agencia ${ }^{6}$. Por esta razón se han tomado como coordenadas de tal ejercicio, por un lado, la

${ }^{4}$ Hay referencia bibliográfica para tres de las investigaciones: Carcar et al. (2020) para el estudio de FLACSO Argentina; Castillo Valencia et al. (próxima publicación) para la investigación de la Universidad del Valle; y FUSALMO (2020) para el estudio de esta institución.

${ }^{5}$ Esta segunda característica se relativiza en el estudio de Guanajuato, donde hay un gran dinamisno económico regional.

${ }^{6}$ Respecto a esta dimensión analítica seguimos la propuesta de Mora Salas y de Oliveira (2014) de «agencia constreñida». En nuestro caso, las constricciones remiten a las violencias presentes y a las pocas oportunidades laborales. 
exposición a la violencia ${ }^{7} \mathrm{y}$, por otro lado, la disposición laboral en el sentido de inserción en el mercado de trabajo. La primera implica que, si bien el entorno es de alta violencia, la persona joven puede desplegar estrategias para reducir tal exposición internalizando y sabiendo manejar los códigos de paralegalidad que impone el actor violento que controla el territorio. La segunda expresa que, si bien las oportunidades son escasas en el mercado de trabajo, hay algunas a las que se pueden acceder, pero para ello hay que tener disposición. No obstante, este último término no sería sinónimo ni de «empleabilidad» ni de «emprendedurismo» porque se trata de agencia condicionada por la escasez de oportunidades laborales remuneradas ${ }^{8}$. Es decir, se quiere destacar la idea de que jóvenes en estas situaciones adversas no permanecen, necesariamente, inermes a las mismas.

Tomando en cuenta estas dos coordenadas, el siguiente gráfico muestra cuatro escenarios para la agencia de jóvenes que corresponden a los cuatro cuadrantes de este sistema de ejes. En cada uno de ellos hay un par de términos que consideramos claves en la caracterización del respectivo escenario. Veámoslos por separado, comenzando con el primer cuadrante y finalizando con el cuarto.

${ }^{7}$ Nos alineamos con la propuesta de Hernández (2001), para quien la violencia contiene tres dimensiones. La primera tiene que ver con hechos de violencia que se expresan en sus efectos humanos (personas muertas, heridas, violadas, refugiadas) o materiales (daños a la propiedad). Una segunda tiene una naturaleza cultural y remite a un espacio de carácter síquico donde se configuran actitudes, conocimientos, emociones, así como representaciones, mitos e ideologías que otorgan significado positivo o negativo a esos hechos. Y hay una tercera dimensión configurada por un conjunto de contradicciones y conflictos sustentados en estructuras sociales y sistemas culturales.

8 Estos términos reflejan la mirada (neo)liberal sobre el mercado laboral: la «empleabilidad» en relación con el trabajo asalariado y el «emprendedurismo» respecto del trabajo no asalariado. Ambos magnifican al individuo y sus capacidades, normalmente centradas en el denominado «capital humano». De esta manera se minimizan los condicionantes situacionales y estos términos se constituyen en imperativos éticos en tanto que se enfatizan los deberes de las/los trabajadoras/es sobre sus derechos. 
GRÁFico 1. EsCENARIOS DE AGENCIA DE JÓVENES DE SECTORES POPULARES URBANOS

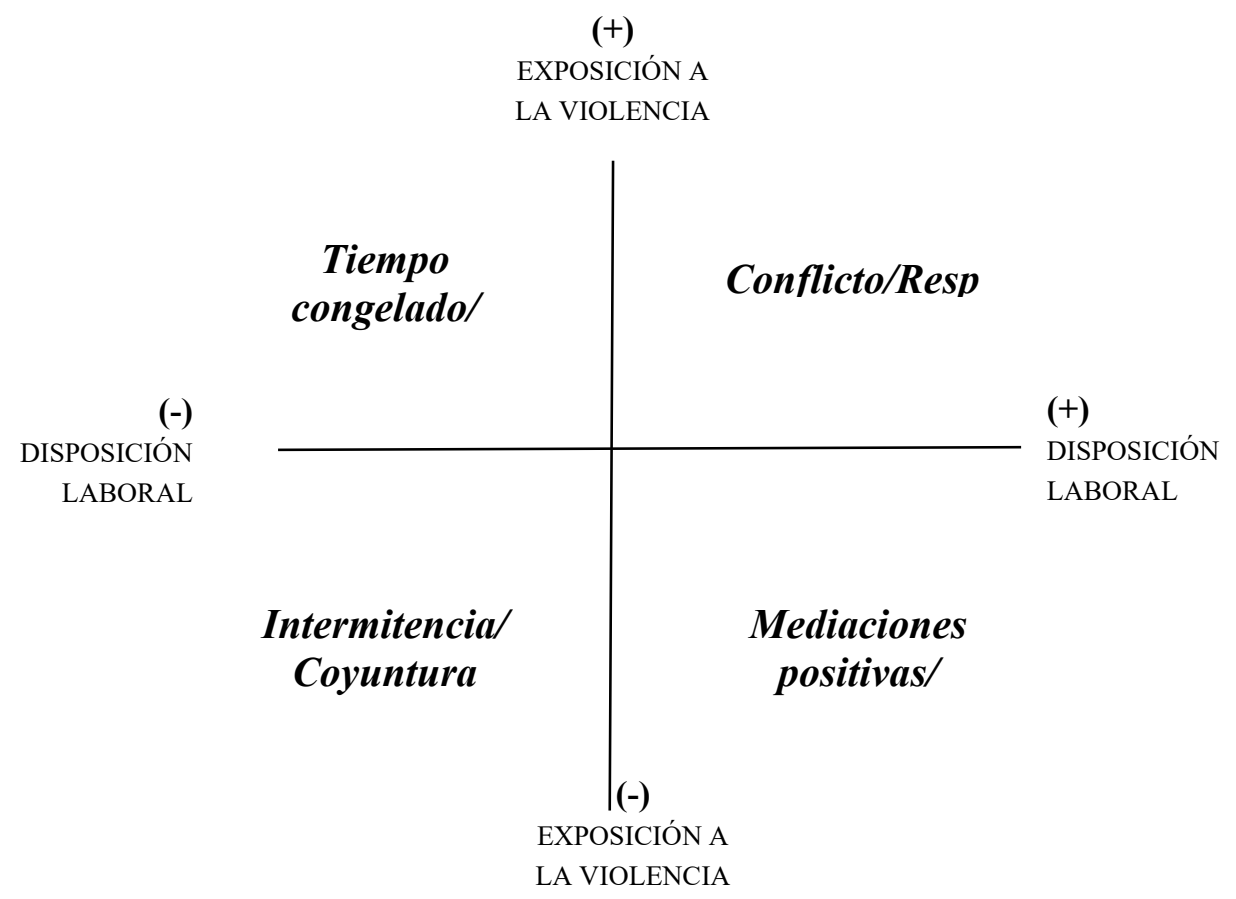

\subsection{EL ESCENARIO DEL DILEMA}

El primero implica situaciones donde la persona joven ha conseguido acceder a una de las pocas oportunidades laborales, sea esta a través de la obtención de un trabajo asalariado o de la autogeneración de una actividad económica, pero sigue expuesta a violencias que ponen en riesgo el logro laboral. Es un escenario de dilemas porque entran en conflicto los dos ejes y su resolución dependerá de la respuesta que logre articular la persona joven. Así, si consigue manejar la amenaza de la violencia podrá consolidar su inserción laboral; de lo contrario, se podría ver engullida por la violencia contextual.

Los estudios mencionados nos ofrecen evidencia empírica al respecto. En el primero de ellos - el realizado por FLACSO en Costa Rica y El Salvadorse entrevistó a un joven salvadoreño que logró abrir un pequeño negocio de fotocopias en su propia colonia. No tardó en recibir la visita de pandilleros que 
comenzaron a extorsionarlo de manera repetida. Este es un fenómeno no limitado al país centroamericano, porque los pobladores de territorios controlados por actores violentos suelen pagarles «tributos»: «peajes» por entrar y salir de ese territorio o transitar dentro de él o extorsiones por ejercer actividades económicas ligadas a ese territorio (pulperías, buses, etc.). Un estudio sobre extorsiones a «micro» y pequeñas empresas en El Salvador revela que este fenómeno es más probable en contextos con presencia de pandillas violentas. Habría extorsiones sistemáticas con pagos periódicos y extorsiones oportunistas, con pago único, que se aprovechan de la existencia del primer tipo de extorsiones. Un hallazgo importante es que en caso de extorsiones sistemáticas estas no siempre son «simbióticas», en el sentido de ofrecer protección a cambio del pago, sino que pueden ser «parasíticas» y — de hecho- son las que predominan (Ponce et al., 2016). Este fue el tipo de extorsión a la que fue sometido este joven salvadoreño y que concluyó con el cierre del negocio por su inviabilidad.

Este fenómeno del «parasitismo» es susceptible de relacionarse con la problemática de la significación del tiempo en contextos de alta violencia, que se abordará en relación con el siguiente escenario. En este sentido, este tipo de extorsión, si bien expresa violencia ganancial, tiende a depredar la fuente de su beneficio y, por tanto, acaba cuestionando ese carácter ganancial reafirmando que, en el caso de pandillas violentas no subordinadas al crimen organizado, su violencia tiene primordialmente una finalidad social en tanto que busca reconocimiento y prestigio.

Otro ejemplo a tomar en cuenta es el fenómeno de los préstamos «gota a gota» mencionado por el estudio de la Universidad del Valle y que no se limita a Cali. Las dificultades de acceso a financiamiento formal es uno de los nueve «factores de desventaja» que condicionan la inserción laboral de jóvenes de sectores populares urbanos que este proyecto ha identificado. Además del ya mencionado, se destacan los siguientes: nivel educativo, condición étnico-racial, género, entorno y cotidianeidad de la violencia, capital social limitado, 
persistencia de patrones de inequidad espacial y estigmatización por lugar de residencia, vínculos previos con pandillas y oferta laboral permanente en actividades ilegales. Este tipo de financiamiento es — en muchos casos - el único accesible para jóvenes de esta extracción social que quieren iniciar o desarrollar un negocio propio. El problema reside en el tipo de garantías: la propiedad de la persona deudora, si la tiene, y en última instancia su propia vida. Es decir, la exposición a la violencia puede devenir máxima.

Finalmente, otro ejemplo que mencionar sería el de algunos de los jóvenes del estudio de FLACSO Argentina que se debaten entre el trabajo en alguno de los proyectos comunitarios y el narcomenudeo. Esta investigación se ha focalizado en población joven marginada y que ha sido víctima de la crisis de la sociedad argentina de finales del siglo pasado e inicio del presente. Esta crisis, junto a la ampliación del mercado de drogas hacia países como Argentina y Uruguay, tuvo un doble efecto sobre el mundo de la marginación del Gran Buenos Aires. Por un lado, incrementó la demanda de «paco», conocida como «la droga de los pobres». Por otro lado, indujo a personas sin trabajo a encontrar en su procesamiento y comercialización un modo de sobrevivir, incrementando las actividades delictivas, lo que implicó crecimiento de la población carcelaria, pero también de muertes. Como respuesta a ello se desarrollaron los Centros Barriales, que se iniciaron como espacios de sociabilidad para esta población afectada por la crisis social.

Una de las estrategias de inserción laboral y de obtención de ingresos que han desarrollado tiene que ver con la puesta de marcha de proyectos socioproductivos, talleres, cooperativas de trabajo o sociales y emprendimientos comunitarios, y otras iniciativas locales implementadas por organizaciones que convocan a los jóvenes. Casi un tercio de los Centros Barriales puso en marcha algunas de estas estrategias centradas inspiradas en el enfoque de economía social y solidaria. El estudio de FLACSO Argentina ha mostrado que, entre quienes no tienen un empleo fuera del emprendimiento, seis de cada diez 
hombres jóvenes no trabajan por problemas asociados al consumo de sustancias y que además les conlleva la realización de actividades ilícitas para la obtención de ingresos.

Por consiguiente, se está ante un escenario donde exposición a la violencia y disponibilidad laboral entran en conflicto, planteando a la persona joven dilemas por resolver que pueden resultar decisivos en su trayectoria de vida.

\subsection{EL ESCENARIO DE LA MUERTE}

Lo primero por destacar en este escenario es que la violencia en su expresión máxima, la letal, forma parte de la cotidianeidad de la persona joven. Esta presencia explícita y permanente de la muerte tiene consecuencias importantes en los referentes temporales y espaciales de la juventud.

En cuanto a los primeros, se puede decir que el tiempo se congela porque lo único que cuenta es el presente. De hecho, este es el tipo de temporalidad propia de la marginación en la que hay que sobrevivir cotidianamente ${ }^{9}$. En este sentido, el futuro se desvanece y, por tanto, las posibilidades de proyectos de vida para las/los jóvenes devienen prácticamente inexistentes. Por otro lado, esta minimización temporal conlleva, como compensación, la maximización espacial. O sea, el territorio deviene todo configurándose en el único referente en tanto que confiere identidad y sentido de vida. Esto es claro en el caso de las pandillas y un/a pandillero/a sin territorio no es nadie. Pero se está ante un referente devorador porque la pertenencia al mismo conlleva conflictos que pueden tener consecuencias letales.

\footnotetext{
${ }^{9}$ Savenije y Andrade-Eekhoff (2003: 194) han argumentado que el abandono estatal es causa de lo que denominan la «temporalidad permanente», que sería el tiempo de la exclusión.
} 
El estudio de la Universidad del Valle es el que mejor ilustra este tipo de escenario. En su estudio de redes se detectó que siete de cada diez de estas/os jóvenes han tenido que lidiar con la muerte de familiares o amigos. Es decir, la muerte violenta forma parte de la cotidianeidad y es percibida como un destino inevitable, en especial en el caso de los hombres, lo cual cuestiona proyectos de vida. El estudio concluye que los jóvenes viven en un «mundo pequeño» donde sus redes personales están conformadas, principalmente, por amigos y familiares. El cierre de este «mundo» tiene implicaciones importantes en términos de violencia porque en este tipo de situaciones las personas jóvenes, ligadas a organizaciones criminales y actividades ilegales, matan a otras/os jóvenes como resultado de conflictos por el dominio territorial, incumplimientos en los negocios, cobro de deudas y narcotráfico ${ }^{10}$.

En este escenario hay una cuestión importante que dilucidar. Ante las escasas oportunidades de trabajo, existe una oferta laboral permanente de actividades ilegales por parte del crimen organizado. Es uno de los nueve «factores de desventaja» que afectan a jóvenes de sectores populares urbanos señalados por el estudio de la Universidad del Valle. Implica actividades tales como el cobro de deudas ligadas a préstamos «gota a gota» o por extorsiones, microtráfico de drogas e incluso la participación directa o indirecta en homicidios selectivos. El 11\% de los jóvenes entrevistados en la información que se recabó sobre redes de ese estudio, expresó abiertamente haber realizado alguna actividad ilegal para generar ingresos en algún momento de su vida.

En este sentido, se podría decir que los jóvenes insertos en tales actividades se desplazarían hacia el primer cuadrante en tanto que habrían tenido

${ }^{10}$ Reguillo (2013) ha identificado cinco circuitos por los que transitan los jóvenes: el de zonas de privilegio, el de la incorporación, el de la asimilación, el de la inviabilidad y el de la «paralegalidad». Este último es el que recorren los jóvenes que han optado por el narcotráfico, la violencia, el crimen organizado, como formas de acceso y afirmación social y correspondería a este escenario. 
acceso a oportunidades laborales. Pensamos que no es así porque en este tipo de actividad es imposible deslindar trabajo de violencia y es esta última la que prevalece. De hecho, el trabajo consiste en ejercer violencia. Esta es una reflexión que remite a las organizaciones delictivas cuya violencia tiene una impronta ganancial. En este sentido, el crimen organizado debe ser considerado como una organización empresarial peculiar y equívoca. De hecho, esta ambigüedad tiene que ver con lo difuso de las fronteras entre lo legal y lo ilegal. Como argumentan Míguez, Misse e Isla (2015), la única diferencia entre empresas legales e ilegales es que estas últimas deben de lidiar — de manera permanente- con su condición de ilegalidad, de ahí el recurso constante a la violencia. Sin embargo, en términos de racionalidad contable y otros recursos legales, no hay diferencias.

En el mismo sentido se podría discutir si se pueden dar proyectos de vida en este escenario y, en concreto, en este tipo de actividades. El perfil de jóvenes participantes en las mismas muestra que la edad de ingreso es entre 8 y 10 años como informantes, para pasar posteriormente a vigilantes alrededor de los 12 años, mientras que ya entre los 14 y 16 años se les premia en la estructura criminal «ascendiéndolos» como sicarios o encargados de un territorio pequeño para venta de droga. Así mismo, puede darse otro tránsito que inicia con el involucramiento de niños como vigías, luego a ser ladrones de casas y asaltantes a transeúntes, para concluir como ladrones de auto y de ahí ser reclutados como «estacas» por las organizaciones criminales, en las que posteriormente pasarán a consolidar su carrera como sicarios (Cisneros, 2014; Encinas Garza, 2016; Ruiz Vázquez et al., 2016). Por consiguiente, existe cierta posibilidad de movilidad interna y de escalamiento de posiciones. Pero a lo largo de estos itinerarios lo harán en posiciones de gran vulnerabilidad, con consecuencias letales altamente probables. En este sentido, es dudoso que estas trayectorias expresen proyectos de vida cuando la muerte deviene tan cercana. 


\subsection{EL ESCENARIO DEL LETARGO}

El cuadrante tercero se caracteriza por la intermitencia entre la obtención de trabajo y el desempleo, pero también de transgresiones ocasionales. En este sentido, predomina la coyuntura, pero no es necesariamente un tiempo congelado, como en el escenario precedente, sino configurado por la sucesión de presentes que no acaban por configurar un futuro. De ahí que lo califiquemos de escenario del letargo.

Los estudios de referencia nos proveen varios ejemplos. En el primero de ellos, el realizado por FLACSO en Costa Rica y El Salvador, una de las cuatro trayectorias identificadas fue la que se denominó «familiar/doméstica» que afectaba a mujeres jóvenes y que suponía su reclusión en el espacio doméstico, reflejando cómo las visiones tradicionales de la división sexual del trabajo tienen aún un fuerte anclaje en el mundo popular. En el caso salvadoreño implicaba protección respecto de la violencia de las pandillas porque la vivienda con su entorno espacial inmediato se considera un «hábitat de seguridad». Es decir, se logra controlar la exposición a la violencia, pero las opciones laborales remuneradas, como las educativas, desaparecen, a pesar de que la gran mayoría de estas mujeres jóvenes, tanto en el caso salvadoreño como costarricense, si tuvieran condiciones favorables les gustaría estudiar o trabajar para obtener un ingreso. La lógica familiar se impone a los deseos de la mujer joven, impidiéndole tener un proyecto de vida autónomo. En el mismo sentido, se puede señalar la evidencia encontrada en el estudio del MMFD en Guanajuato. Las familias han limitado la participación de las hijas para que no opten por carreras técnicas calificadas como masculinas. Además, cuando la estudiante aspira a ingresar al MMFD, los planteles educativos no tienen una política institucional que aliente tal participación. Si logran superar estos obstáculos y son aceptadas en el programa, los progenitores argumentan la existencia de acoso en las firmas para desmotivarlas e impedir que participen en el MMFD. De ahí que sea una experiencia con gran sesgo de masculinidad. 
La investigación de FLACSO Argentina muestra trayectorias laborales signadas por trabajos precarios, por sus condiciones de contratación y por la baja tasa de empleo registrado, y largos períodos de inactividad. La estabilidad laboral es prácticamente inexistente y los momentos de ocupación son cortos. Como se argumenta en este estudio, a pesar del inicio prematuro en el mercado laboral, las trayectorias han estado entrecortadas con períodos prolongados de inactividad. Es decir, ha sido muy difícil para estas/os jóvenes lograr trayectorias laborales que acumulen experiencias que los fortalezca en el mercado de trabajo.

Finalmente, el estudio de FUSADES, centrado sobre el importante tema de la estigmatización, muestra cómo ciertas familias evitan que sus hijos/as busquen trabajo fuera de la colonia por estar marcados por su pertenencia territorial. De esta manera internalizan tal estigma y se configuran en jóvenes que el estudio caracteriza como «autolimitados», que no se atreven salir de la comunidad en busca de trabajo y quedan atrapados en la misma.

La problemática de la estigmatización de jóvenes de sectores populares está presente en todos los escenarios, pero sería en este tercero que emerge de manera más nítida. Ya se señaló que es uno de los factores críticos de articulación entre violencias y mundo de trabajo para las/los jóvenes (Mora Salas y Pérez Sáinz, 2018). Inicialmente se trata de la estigmatización del territorio resultante de una metonimia que atribuye la naturaleza violenta del actor que controla el territorio a todos los pobladores, y en especial a las personas jóvenes, por compartir ese espacio. Se ha señalado en Santiago de Chile, fruto del reordenamiento territorial de esta ciudad bajo la dictadura pinochetista, respecto de ciertas comunas donde predominan sectores subalternos. Así, estos son estigmatizados a través de una relación metonímica entre quienes realizan delitos y el territorio que habitan. Desde las percepciones predominantes, especialmente a través de los medios de comunicación, se homogeniza socialmente al territorio y todos sus pobladores devienen delincuentes (Cornejo, 2012). Esta metonimia es también clara en el caso de los países septentrionales de Centroamérica, donde 
se tiende a visualizar a los hombres jóvenes de asentamientos marginales como pandilleros, estigmatizándolos (Aguilar Umaña, 2016).

Este fenómeno limita las oportunidades laborales por la cultura de estigmatización que se impone en la sociedad y, en especial, en los empresarios. Es decir, estos son reacios a contratar personas jóvenes, especialmente hombres, que residen en territorios caracterizados como violentos. Aquí el gran reto no es la desestigmatización individual, que se puede lograr a través de programas de formación, sino la colectiva, o sea, la del propio territorio.

Pero la estigmatización no se reduce a lo territorial. Los estudios de referencia muestran que el mero hecho de ser joven conlleva también marca ${ }^{11}$, como muestra la investigación de FUSADES, al igual que la condición afrodescendiente en Cali o los antecedentes penales en el Gran Buenos Aires. Es decir, la inserción laboral de estas/os jóvenes se ve entrabada por este conjunto de estigmas.

\subsection{EL ESCENARIO DE LA VIDA}

Este último escenario es el opuesto al segundo, de ahí que lo califiquemos — por antítesis - como escenario de la vida. La violencia se minimiza, lo cual no implica que el territorio deja de ser violento, pero la persona joven internaliza y maneja hábilmente los códigos de la paralegalidad a su favor. Por otro lado, ha logrado una inserción no precaria en el mercado de trabajo, es decir, ha sabido

${ }^{11}$ Hace tiempo, Perea Restrepo (2005) desmontó ese estigma, a partir del análisis de datos sobre las dos últimas décadas del siglo pasado en México respecto a una serie de delitos (homicidios, robo y delitos contra la salud), comparando distintos grupos etarios. A partir de esta evidencia, este autor concluye que el estigma no tiene que ver con la magnitud de la participación delictiva de los jóvenes, sino más bien con sus modalidades. Al respecto, sobresalen dos que han adquirido en los últimos tiempos gran significación simbólica: las pandillas, especialmente las violentas, y el sicariato. Pandillero y sicario son las figuras, especialmente masculinas, que nutren el miedo social erigiendo la amenaza de la «violencia juvenil». 
aprovechar una de las pocas oportunidades laborales existentes. Esto le provee un referente para pensar su futuro y esbozar un proyecto de vida.

En dos de los estudios se insinúa este escenario. En la mayoría de las/los jóvenes de la investigación de FUSALMO se detectan diferentes tipos de aspiraciones: estudios, compra de propiedad mueble y/o inmueble, formación de familia, etc. Se argumenta que las/los jóvenes han visualizado un proyecto de vida a partir del empleo no precario, al contrario de experiencias laborales anteriores, que han obtenido gracias a la intermediación laboral de FUSALMO. Al respecto es importante destacar que, salvo contadas excepciones, estas/os jóvenes no se plantean la emigración. Es decir, el «sueño americano», tan presente en la juventud salvadoreña (Gaborit et al., 2012), no aparece en su horizonte, se lucha por conseguir un «sueño salvadoreño».

También este escenario emerge en el estudio de Guanajuato. Se ha enfatizado que la participación de jóvenes en el MMFD conllevaría cuatro resultados. Primero se verían beneficiados de una formación integral, tanto teórica como práctica, lo cual les provee ventajas con sus compañeros de los planteles educativos que no han tomado el programa. Segundo, los aprendices duales se ven favorecidos por el abanico de opciones al concluir el programa: pueden ser contratados por la empresa y continuar o no con sus estudios; dedicarse únicamente a estos realizando estudios universitarios; o entrar en el mercado de trabajo con ventajas comparativas respecto a mano de obra formada de manera tradicional. Tercero, estas opciones insinúan posibilidades de movilidad social, por representar mejores ocupaciones que las de sus padres. Y, finalmente, se crearían condiciones para alejar a estos jóvenes de contextos violentos, sean familiares o barriales.

No obstante, hay que ser precavidos y no caer en la tentación de que se está ante jóvenes que habrían logrado la quimera de la «empleabilidad», que les 
permitiría superar cualquier escollo laboral ${ }^{12}$. Así, el estudio de FUSALMO es enfático al señalar que, pese al seguimiento institucional de las/os jóvenes, una vez que son empleada/os quedan en manos de los empresarios o jefes inmediatos, que no siempre están dispuestos a apoyarles. Y en el caso de Guanajuato, no se puede ignorar el contexto de gran dinamismo económico regional de los últimos años.

En la configuración de este escenario, como en los otros, juegan un papel clave las mediaciones (familia, escuela, trabajo, instituciones, territorio y pares) que explicarían comportamientos diferenciados de los jóvenes (Espíndola Ferrer, 2013). Su consideración en el análisis permite evitar tanto el determinismo estructural (todas/os las/os jóvenes en situación de marginación social tienen un mismo destino) como el voluntarismo individual (sí se puede porque hay suficientes oportunidades).

Estas mediaciones pueden contribuir a que la persona joven logre esbozar un proyecto de vida potenciando su agencia, como también puede obstruirlo. Implican ámbitos de interacción social con dinámicas de distinto signo. Los estudios de referencia muestran ejemplos. Lo familiar y lo escolar emergen como referentes que refuerzan la perspectiva de un proyecto de vida en las/los jóvenes de la investigación de FUSALMO. Lo familiar es el primer y principal filtro para la participación en el MFDD en Guanajuato mostrando, justamente, su doble cara: incentiva a los hombres, pero inhibe a las mujeres. Y en el caso del estudio de FLACSO Argentina se reivindica lo territorial a través de las redes comunitarias, producto de las organizaciones barriales. Han servido para empezar a recomponer las trayectorias rotas de las personas jóvenes de los barrios

${ }^{12}$ Hay que recordar que la problemática de la empleabilidad se constituye a partir del señalamiento institucional de supuestos déficits familiares en términos de trayectos, aprendizajes y saberes (Assusa, 2017). Representa una auténtica estrategia de nuevo disciplinamiento de fuerza laboral joven, en concreto en su componente de «habilidades blandas». 
marginalizados del Gran Buenos Aires, generando nuevas gramáticas juveniles, apelando a la vida solidaria y a la construcción de grupos familiares diversos. Pero ha sido el primer estudio de FLACSO el que ha ahondado más en esta problemática de las mediaciones. A partir de estudios de caso tanto en jóvenes salvadoreños como costarricenses se identificaron cuatro tipos de vías laborales: profesionalizante, vulnerable, precaria y familiar/doméstica. Solo en la primera, que se inscribiría en el presente escenario, se pudo percibir una trayectoria de salida de la condición de marginación gracias a que la mayoría de las mediaciones se alinearon de manera positiva, generando una dinámica acumulativa y virtuosa. Desgraciadamente, fueron muy pocas las personas jóvenes, en ambos universos, que la pudieron transitar.

\section{CONCLUSIONES}

Como se ha mencionado en la introducción, se quiere concluir con algunas reflexiones preliminares sobre la incidencia de la actual pandemia sobre estos escenarios ${ }^{13}$.

La pandemia, en sus inicios, ha afectado al mercado laboral en América Latina de múltiples maneras ${ }^{14}$. Ha habido un importante descenso de la tasa de ocupación, que no se reflejó con la misma magnitud en la tasa de desempleo abierto porque parte de la fuerza laboral desplazada no buscó trabajo ante las

${ }^{13}$ Hay dos encuestas, a nivel mundial, sobre la incidencia de la pandemia en jóvenes. La primera ha sido llevada a cabo por la OIT (2020) en 112 países, con más de 12.000 respuestas provenientes de jóvenes, entre 19 y 29 años, instruidos y con acceso a internet, por lo que el grupo con educación superior es el único representativo. La segunda encuesta es la realizada por Plan International (2020) en 14 países a mujeres jóvenes y adolescentes (15 a 24 años), con una muestra de un poco más de 7.000 jóvenes.

${ }^{14}$ La evidencia proviene de información estadística de Brasil, Chile, Colombia, México y Perú (limitado a Lima Metropolitana) del primer semestre del año (Weller et al., 2020). 
oportunidades mínimas y las imposiciones de confinamiento. El trabajo no asalariado, en concreto el por cuenta propia, se ha visto más afectado que el asalariado. Construcción, y alojamiento y restaurantes han sido las ramas más perjudicadas. También el impacto ha sido mayor en el sector privado que en el público. La jornada laboral se ha reducido, impactando en los ingresos. Este conjunto de rasgos insinúa que los efectos han sido mayores en la fuerza de trabajo más vulnerable, que - con menor calificación- ha tenido menos posibilidades de recurrir al teletrabajo (Weller et al., 2020). Es decir, serían los sectores populares los más afectados. En efecto, siete de cada diez hogares del quintil inferior de ingreso habrían sufrido la pérdida de al menos un empleo mientras en el quintil superior habrían sido dos de cada diez hogares (Busso y Messina, 2020: 53). Dentro de los sectores populares, las personas jóvenes padecen más estos efectos negativos, especialmente la fuerza de trabajo entrante $^{15}$. Con el gradual abandono del confinamiento por los imperativos económicos, estas tendencias deben haber tenido alguna transformación, pero en un contexto de profunda recesión.

Los impactos negativos sobre el mercado de trabajo se reflejarían en el gráfico propuesto de escenarios por el desplazamiento del eje de ordenadas hacia la derecha, reduciendo las áreas del primero y del cuarto cuadrantes, o sea, las oportunidades laborales serían aún menores.

La pandemia ha abierto una oportunidad para que el Estado recupere presencia en territorios marginados. Los gobiernos de la región han implementado medidas de protección a la población más vulnerable. Algunas como la prohibición de desconexión de servicios básicos de la vivienda por

${ }^{15}$ La mencionada encuesta mundial de la OIT (2020: 15-24) muestra que el $17 \%$ de las personas jóvenes que estaban trabajando antes de la pandemia, perdieron su trabajo. La jornada laboral se redujo casi a una cuarta parte y $42 \%$ sufrieron reducción en sus ingresos laborales, fenómenos que se acentúan en países de bajos ingresos (OIT, 2020: $15-24)$. 
incumplimiento de pagos, no pago del alquiler de la vivienda o la distribución de alimentos han implicado presencia estatal en el mundo de la marginación social. Pero este vínculo territorial no ha sido el principal. Por el contrario, se ha privilegiado un nexo monetario a través de transferencias en dinero, aprovechando la infraestructura institucional generada por los programas de transferencias condicionadas.

La otra cara de la moneda es qué ha pasado con los actores violentos que controlan esos territorios. No hay que olvidar que, desde antes de la pandemia, ha intentado legitimar su orden paralegal con la oferta de una serie de bienes comunitarios: protección respecto a agresiones externas; intermediación de conflictos intracomunitarios, sean domésticos o entre vecinos; mediación de la actividad comunitaria, filtrando y permitiendo acciones de agentes e instituciones extracomunitarias; y celebración de actividades festivas que recrean la comunidad y que son muy valoradas por los pobladores, especialmente por jóvenes (Perea et al., 2014). Habría que ver si han ampliado tal oferta para ayudar a los pobladores a afrontar la pandemia. Además, dada la pérdida de dinamismo del mercado de trabajo, la oferta laboral permanente del crimen organizado ganaría relevancia.

Es decir, en este caso no se puede señalar si ha habido desplazamiento del eje de abscisas y habría que constatarlo en cada situación concreta.

Además de estos dos grandes referentes situacionales, hay que plantearse interrogantes sobre el propio actuar de las/los jóvenes.

Lo primero sería indagar los efectos del confinamiento sobre el comportamiento juvenil en varios ámbitos. Así, el espacio se ha reducido a la vivienda o, en el mejor de los casos, al entorno comunitario. Habría que ver cuál uso de estos espacios han hecho las personas jóvenes, poniendo especial atención a que, en situación de confinamiento, se habrían incrementado las probabilidades de interacción con actores violentos que controlan el territorio. Sin embargo, esta 
dimensión no se limita al espacio físico, sino que los virtuales han ganado relevancia. Determinar su impacto sería importante. Otro ámbito es lo familiar, que plantea cuestionamientos en un doble sentido: por un lado, estarían las dinámicas de sociabilidad al interior del grupo familiar que deberían haberse intensificado; pero, por otro lado, no se pueden obviar momentos de tensión y conflicto con posibles secuelas de violencia intradoméstica, donde mujeres y menores suelen ser víctimas. Y el tercer ámbito sería el de la reproducción material del hogar y la contribución que han hecho al respecto los miembros jóvenes. Esta es una contribución que no se limita a la obtención de ingresos sino también de ayudas, a través de instituciones y redes de distinto tipo. Y, por supuesto, de gran importancia es la realización del trabajo doméstico, donde la cuestión de género deviene central. Al respecto, el tema de cuidados se convierte en sustancial, sobre todo si hay menores y/o personas con capacidades diferentes y si además ha habido miembros del hogar afectados por el virus.

Un segundo conjunto de interrogantes tiene que ver con las acciones tomadas por jóvenes ante el relajamiento de medidas de confinamiento y, en concreto, respecto de la inserción al mercado de trabajo. Así, en el caso de recuperación de empleos anterior, habría que indagar si ha habido cambios en las condiciones laborales preexistentes a la pandemia. En cuanto a actividades autogeneradas, las preguntas deben versar sobre su (re)invención en términos de disponibilidad de recursos. Y si la opción es la búsqueda de empleo, habría que conocer cuáles estrategias estarían desplegando las personas jóvenes. En el trasfondo de estas acciones no hay que olvidar la oferta laboral permanente del crimen organizado.

Sus percepciones sobre este momento tan peculiar es otro conjunto de interrogantes, partiendo del supuesto de que la pandemia debe constituir un hito 
relevante en su biografía ${ }^{16}$. Así, ¿cómo se ha vivido el confinamiento? ¿Qué cambios traerá a su vida la pandemia? ¿Cómo visualiza su futuro y qué acciones espera tomar? ¿Se ha visto redefinido su proyecto de vida, en caso de que lo tuviera, y cómo?

Hay un buen número de interrogantes que esbozan problemáticas de investigación de cara al futuro inmediato, para poder entender cómo las personas jóvenes de sectores populares van a poder confrontar la gran crisis que ha planteado la pandemia actual.

ENVIADO: 1 DE FEBRERO DE 2021

ACEPTADO: 14 DE JULIO DE 2021

${ }^{16}$ En la encuesta de la OIT (2020: 36), el 38\% de las/los jóvenes mostraron inseguridad ante sus futuras perspectivas profesionales y el 16\%, temor. En la encuesta de Plan International (2020: 10) el 95\% de las jóvenes señalaron que la pandemia había sido perjudicial para sus vidas por limitar sus posibilidades de educación, de socialización con pares y por la merma de ingresos en el hogar. 


\section{BibLIOGRAFÍA}

Aguilar UMAÑA, I. (2016). Victimarios y víctimas de la violencia: de nexos invisibilizados y falsas dicotomías en el Triángulo Norte de Centroamérica. En M. GOTTSBACHER y J. DE BOER (coords.), Vulnerabilidad y violencia en América Latina. México, D. F.: Siglo $\mathrm{XXI} /$ Proyectos Estratégicos Consultoría.

AssusA, G. (2017). Jóvenes trabajadores: disputas sobre sentidos, apropiaciones simbólicas y distinciones sociales en el mundo laboral. Buenos Aires: Grupo Editor Universitario.

Busso, M. y Messina, J. (eds.) (2020). La crisis de la desigualdad: América Latina y el Caribe en la encrucijada. Washington, D. C.: Banco Interamericano de Desarrollo.

Carcar, F., Vázquez, M., Arancibia, M., Fainstein, C. y Miranda, A. (2020). Trayectorias rotas: resultados de la investigación entre jóvenes pares en Centros Barriales del Gran Buenos Aires. Documento de Trabajo, 3. Disponible en: https://www.flacso.org.ar/wpcontent/uploads/2020/08/Doc.-de-Trabajo-N-3-Informe-investigacionentre-pares.pdf

Castillo VAlencia, M. DEL P. ET AL. (próxima publicación). Las desventajas de ser joven en Cali: pandillas, barreras y mundos pequeños. Cali: Editorial Universidad del Valle.

Castillo Valencia, M. Del P., Salazar Trujillo, B. y Caicedo Hurtado M. I. (2019). Exclusión laboral de jóvenes, pandillas y crimen organizado. Reflexiones desde Cali. En J. P. PÉrEZ SÁInZ (ed.), A golpes de presente, a gritos de futuro. Jóvenes, trabajo y violencias en América Latina. San José: FLACSO Costa Rica.

Cisneros, J. L. (2014). Niños y jóvenes sicarios: una batalla cruzada por la pobreza. El Cotidiano, 186, 7-18.

CORNEJO, C. A. (2012). Estigma territorial como forma de violencia barrial. El caso del sector de El Castillo. Revista Invi, 27(76), 177-200.

CRUZ SiERrA, S. (2019). Jóvenes y construcción de masculinidades en contextos de marginación social y violencia. Reflexiones desde Ciudad Juárez. En J. P. PÉrez SÁinz (ed.), A golpes de presente, a gritos de futuro. Jóvenes, trabajo y violencias en América Latina. San José: FLACSO Costa Rica.

ENCINAS GARZA, J. L. (2016). Jóvenes sicarios. La generación desechable: vivir rápido y morir joven. CiENCiA UANL, 19(80), 59-65.

EsPíNDOLA FERRER, F. (2013). Por una praxis de alternativas: jóvenes montevideanos «rescatando» destinos. Serie Documentos de Trabajo, Red de Posgrados, 39. Buenos Aires: CLACSO. 
FUSALMO. (2020). Trayectorias sociolaborales de jóvenes participantes en el proyecto «Jóvenes creando futuro». Informe de resultados Fase II. Soyapango: FUSALMO. Disponible en: https://fusalmo.org/Sociolaboral/TRAYECTORIASSOCIOLABORALE SDEJOVENES.pdf

Gaborit, M., Zetino Duarte, M., Brioso, L. y Portillo, N. (2012). La esperanza viaja sin visa: jóvenes y migración indocumentada de El Salvador. Serie Investigaciones, 1. San Salvador: FNUAP/UCA.

HERNÁNDEZ, T. (2002). Des-cubriendo violencia. En R. BRICEÑO-LEÓN (comp.), Violencia, sociedad y justicia en América Latina. Buenos Aires: CLACSO.

Hualde Alfaro, A. (2019). Jóvenes en trabajos globalizados: el caso de los call centers en México. En J. P. PÉREZ SÁInZ (ed.), A golpes de presente, a gritos de futuro. Jóvenes, trabajo y violencias en América Latina. San José: FLACSO Costa Rica.

LÓPEZ RAmíreZ, A. R. (2019). Las pandillas como actores de poder en el Triángulo Norte de Centroamérica. En J. P. PÉrEz SÁInZ (ed.), A golpes de presente, a gritos de futuro. Jóvenes, trabajo y violencias en América Latina. San José: FLACSO Costa Rica.

MirANDA RodRígUEZ, A. (2019). Estrategias de emprendimientos productivos y economía social popular para la generación de ingresos: juventudes, territorios y género. En J. P. PÉREZ SÁINZ (ed.), A golpes de presente, a gritos de futuro. Jóvenes, trabajo y violencias en América Latina. San José: FLACSO Costa Rica.

Míguez, D., Misse, M. e Isla, A. (2015). Contingencias en las relaciones entre Estado, Gobierno y crimen organizado en América Latina. En D. MígUEZ, M. MiSSE y A. ISLA (comps.), Estado y crimen organizado en América Latina. Buenos Aires: Araucaria.

Mora Salas, M. y De Oliveira, O. (2014). ¿Ruptura o reproducción de las desventajas sociales heredadas? Relatos de vida de jóvenes que han vivido situaciones de pobreza. En M. MORA SAlas y O. DE OliveIRA (coords.), Desafíos y paradojas. Los jóvenes frente a las desigualdades sociales. México, D. F.: El Colegio de México.

Mora SAlas, M. y PÉRez SÁinz, J. P. (2018). El desafío de inclusión laboral de jóvenes en barrios urbano-marginales en Centroamérica: más allá de las políticas de capacitación para el empleo. En A. CORICA, A. FrEYTES FREY y A. MIRANDA (coords.), Entre la educación y el trabajo: la construcción cotidiana de las desigualdades juveniles en América Latina. Buenos Aires: CLACSO. 
OIT. (2020). Los jóvenes y la Covid-19: efectos en los empleos, la educación, los derechos y el bienestar mental. Informe de la encuesta 2020. Ginebra: OIT.

Perea Restrepo, C. M. (2005). Joven, crimen y estigma. Quórum, 12, 65-94.

Perea, C. M., Jaramillo, A. M., Rincón Morera, A., Misse, M., Alarcón, C. y GIL, M. Y. (2014). Introducción. La paradoja latinoamericana. Las ciudades en perspectiva comparada. En A. M. JARAMILlO y C. M. PEREA (eds.), Ciudades en la encrucijada: Violencia y poder criminal en Río de Janeiro, Medellín, Bogotá y Ciudad Juárez. Medellín: Región/IEPRI/Universidad Nacional de Colombia/IDRC.

PÉrez SÁInZ, J. P. (ed.) (2019). Jóvenes, violencia y trabajo en América Latina. Una propuesta de factores críticos de articulación. En J. P. PÉREZ SÁINZ (ed.), A golpes de presente, a gritos de futuro. Jóvenes, trabajo y violencias en América Latina. San José: FLACSO Costa Rica.

. (2018). Vidas sitiadas. Jóvenes, exclusión laboral y violencia urbana en Centroamérica. San José: FLACSO/IDRC-CDRI.

Plan International. (2020). Vidas detenidas. El impacto de la Covid-19 en niñas y mujeres jóvenes. Disponible en: https://planinternational.org/sites/default/files/vidas_detenidas_covid19vidas_deten idas_070920_vf2.pdf

Ponce, C., Beneke de SAnfeliú, M., ChÁvez, M. y Shi, M. (2016). Extorsiones a la micro y pequeña empresa de El Salvador. Documento Proyecto SolucionES. San Salvador: FUSADES.

REguiLlo, R. (2013). Jóvenes en la encrucijada contemporánea: en busca de un relato de futuro. Debate Feminista, 48, 137-151.

Ruiz Vásquez, A., García Campos, T., Padrós Bláquez, F. y Sahagún PADILlA, Á. (2016). El sicariato: una perspectiva psicosocial del asesinato por encargo. Revista Electrónica de Psicología Iztacala, 19(3), 994-1013.

SaveniJe, W. y Andrade-Eekhoff, K. (2003). Conviviendo en la orilla. Violencia y exclusión en el Área Metropolitana de San Salvador. San Salvador: FLACSO.

Weller, J., Gómez Contreras, M., Martín Caballero, Á. y Ravest Tropa, J. (2020). El impacto de la crisis sanitaria del COVID-19 en los mercados laborales latinoamericanos. Documentos de Proyectos (LC/TS.2020/90). Santiago de Chile: CEPAL. 